\title{
Statins Attenuate Activation of the NLRP3 Inflammasome by Oxidized LDL or TNF $\alpha$ in Vascular Endothelial Cells through a PXR-Dependent Mechanism $\$$
}

\author{
Shaolan Wang, Xinya Xie, Ting Lei, Kang Zhang, Baochang Lai, Zihui Zhang, Youfei Guan, \\ Guangmei Mao, Lei Xiao, and Nanping Wang \\ Cardiovascular Research Center, School of Medicine, Xi'an Jiaotong University, Xi'an, People's Republic of China (S.W., X.X., \\ T.L., K.Z., B.L., Z.Z., L.X., N.W.); The Advanced Institute for Medical Sciences, Dalian Medical University, Dalian, People's \\ Republic of China (Y.G., N.W.); and Department of Quantitative Health Sciences, Cleveland Clinic, Cleveland, Ohio (G.M.) \\ Received December 25, 2016; accepted May 22, 2017
}

\begin{abstract}
Excessive activation of the NLRP3 inflammasome is implicated in cardiovascular diseases. Statins exert an anti-inflammatory effect independent of their cholesterol-lowering effect. This study investigated the potential role of statins in the activation of the NLRP3 inflammasome in endothelial cells (ECs). Western blotting and quantitative reverse-transcription polymerase chain reaction showed that oxidized low-density lipoprotein (ox-LDL) or tumor necrosis factor $\alpha$ (TNF $\alpha$ ) activated the NLRP3 inflammasome in ECs. Simvastatin or mevastatin significantly suppressed the effects of ox-LDL or TNF $\alpha$. Promoter reporter assays and small interfering RNA knockdown revealed that statins inhibit ox-LDL-mediated NLRP3 inflammasome activation via the pregnane $X$ receptor (PXR). In addition, PXR agonists (rifampicin and
\end{abstract}

SR12813) or overexpression of a constitutively active PXR markedly suppressed the NLRP3 inflammasome activation. Conversely, PXR knockdown abrogated the suppressive effect of rifampicin on NLRP3 inflammasome activation. Knockdown of lectin-like ox-LDL receptor or overexpression of $I_{\kappa} \mathrm{B} \alpha$-attenuated ox-LDL- or TNF $\alpha$-triggered activation of the NLRP3 inflammasome. Chromatin immunoprecipitation assays indicated that mevastatin inhibited nuclear factor- $\mathrm{\kappa}$ binding to the promoter regions of the human NLRP3 gene. Collectively, these results demonstrate that the statin activation of PXR inhibits the activation of NLRP3 inflammasome in response to atherogenic stimuli such as ox-LDL and TNF $\alpha$ in ECs, providing a new mechanism for the cardiovascular benefit of statins.

\section{Introduction}

Endothelial cells (ECs) exert critical functions in maintaining homeostasis in the vascular system. Endothelial inflammation is a major contributor to endothelial dysfunction in cardiovascular disorders such as hypertension, diabetes, and atherosclerosis (Davignon and Ganz, 2004). Interleukin (IL)- $1 \beta$ is a key proinflammatory mediator involved in endothelial dysfunction and serves as a major atherogenic factor (Fearon and Fearon, 2008). The production of active IL-1 $\beta$ is tightly controlled through the formation and activation of

This study was supported by grants from the National Science Foundation of China [Grants NSFC 31430045, 30881220108005, and 81470373].

https://doi.org/10.1124/mol.116.108100.

S This article has supplemental material available at molpharm.aspetjournals. org. the inflammasome. The NLRP3 inflammasome, the bestcharacterized family member, is a multiprotein complex consisting of NLRP3, caspase-1, and the adaptor ASC (PYCARD). Activation of the NLRP3 inflammasome promotes the cleavage of caspase- 1 and the maturation of IL- $1 \beta$ and IL-18. Excessive activation of the NLRP3 inflammasome and the ensuing IL- $1 \beta$ secretion have recently emerged as central mechanisms in the pathogenesis of metabolic diseases, including type II diabetes, obesity, and atherosclerosis (Mitroulis et al., 2010; Strowig et al., 2012). Activation of NLRP3 in ECs, triggered by dyslipidemia, disturbed blood flow, and visfatin, may contribute to the development of atherosclerosis and restenosis (Xia et al., 2014; Xiao et al., 2013).

Statins comprise a class of cholesterol-lowering drugs widely used in the treatment of hypercholesterolemia and

ABBREVIATIONS: Ad, adenovirus; Ad-VP-PXR, adenovirus encoding constitutively active pregnane $\mathrm{X}$ receptor; BAEC, bovine aortic endothelial cell; CAD, coronary artery disease; ChIP, chromatin immunoprecipitation; EC, endothelial cell; HUVEC, human umbilical vein endothelial cell; IL, interleukin; LDL, low-density lipoprotein; LOX-1, lectin-like oxidized low-density lipoprotein receptor; Luc, luciferase; NF- $\kappa$ B, nuclear factor- $\kappa B$; ox-LDL, oxidized-low-density lipoprotein; PXR, pregnane X receptor; PXRE, PXR-responsive element; qRT-PCR, quantitative reverse-transcription polymerase chain reaction; ROS, reactive oxygen species; siRNA, small interfering RNA; TNF $\alpha$, tumor necrosis factor $\alpha$; tTA, tetracyclineresponsive transactivator; VP-PXR, constitutively active pregnane $\mathrm{X}$ receptor. 
TABLE 1

Primers for ChIP assay

\begin{tabular}{cll}
\hline Primer & \multicolumn{1}{c}{ Forward $\left(5^{\prime}\right.$ to $\left.3^{\prime}\right)$} & \multicolumn{1}{c}{ Reverse $\left(5^{\prime}\right.$ to $\left.3^{\prime}\right)$} \\
\hline-847 to -838 & CAAGGAGGGGGCAATTCCAA & TGAAAAGGACGAGCACCCAG \\
-166 to -157 & ACTCGCATGGCATGTCCTTA & TCTCGGTTCCATACTGGGGT \\
-110 to -101 & ACCGAGACACGGTTTTGAC & GCTGGACTTACCCAGATGCC \\
\hline
\end{tabular}

coronary artery diseases (CADs). Acting as 3-hydroxy-3methylglutaryl CoA reductase inhibitors, statins increase the expression of low-density lipoprotein (LDL) receptor in the liver, resulting in increased cholesterol uptake and lowered circulating cholesterol levels. In addition, statins have immunomodulatory and anti-inflammatory properties (Spyridopoulos et al., 2004; Morimoto et al., 2006). Statins also improve endothelial function (Kesavan et al., 2014). Recent studies have shown that statins affect the anti-NLRP3 in patients with cardiovascular disease. A randomized clinical study showed that atorvastatin markedly diminished NLRP3 inflammasome levels in CAD patients (Satoh et al., 2014). In monocytic THP-1 cells, atorvastatin inhibited activation of the NLRP3 inflammasome (Satoh et al., 2014). Rosuvastatin has been reported (Luo et al., 2014) to alleviate diabetic cardiomyopathy by inhibiting NLRP3 inflammasome and mitogenactivated protein kinase pathways in a rat model of type II diabetes. However, the mechanisms by which statins regulate NLRP3 inflammasome remain largely unknown.

The nuclear pregnane $\mathrm{X}$ receptor [PXR (NR1I2)] is a member of the nuclear receptor superfamily and functions as the key regulator of the body's defense against xenobiotics, including pollutants, drugs, dietary compounds, and their metabolites (Kliewer et al., 2002). In addition to its conventional role in xenobiotic metabolism in liver and intestines, we

and others (Wang et al., 2013) also found that PXR is expressed in the vasculature, where it regulates vascular detoxification and inflammation. In particular, PXR suppresses the expression of proinflammatory adhesion molecules, such as vascular cell adhesion molecule- 1 and endothelial selectin in response to $\mathrm{TNF} \alpha$ and lipopolysaccharide in ECs. Overexpression of a constitutively active PXR (VP-PXR) in rat carotid arteries attenuated proinflammatory responses (Wang et al., 2013). Notably, PXR appears to orchestrate the regulated expression of the key genes responsible for detoxification and innate immunity programs in ECs (Wang et al., 2014). Statins have been known to activate PXRs (Howe et al., 2011). Thus, we sought to investigate the effects of statins in the activation of NLRP3 inflammasomes in ECs and the underlying mechanisms.

\section{Materials and Methods}

Cells and Reagents. Human umbilical vein ECs (HUVECs) were cultured in medium 199 containing heparin $(0.1 \mathrm{mg} / \mathrm{ml})$, acidic fibroblast growth factor $(10 \mathrm{ng} / \mathrm{ml})$, L-glutamine $(2 \mathrm{mM})$, penicillin $(100 \mathrm{U} / \mathrm{ml})$, streptomycin $(100 \mathrm{U} / \mathrm{ml})$, and $20 \%$ fetal bovine serum. Bovine aortic ECs (BAECs) were maintained in Dulbecco's modified Eagle's medium with $10 \%$ fetal bovine serum, penicillin $(100 \mathrm{U} / \mathrm{ml})$, and streptomycin (100 U/ml). Rifampicin was from Cayman (Ann Arbor, MI). Antibodies against NLRP3 and nuclear factor- $\kappa \mathrm{B}(\mathrm{NF}-\kappa \mathrm{B})$ p65 were from Abcam (Cambridge, UK). Antibody to caspase-1 was
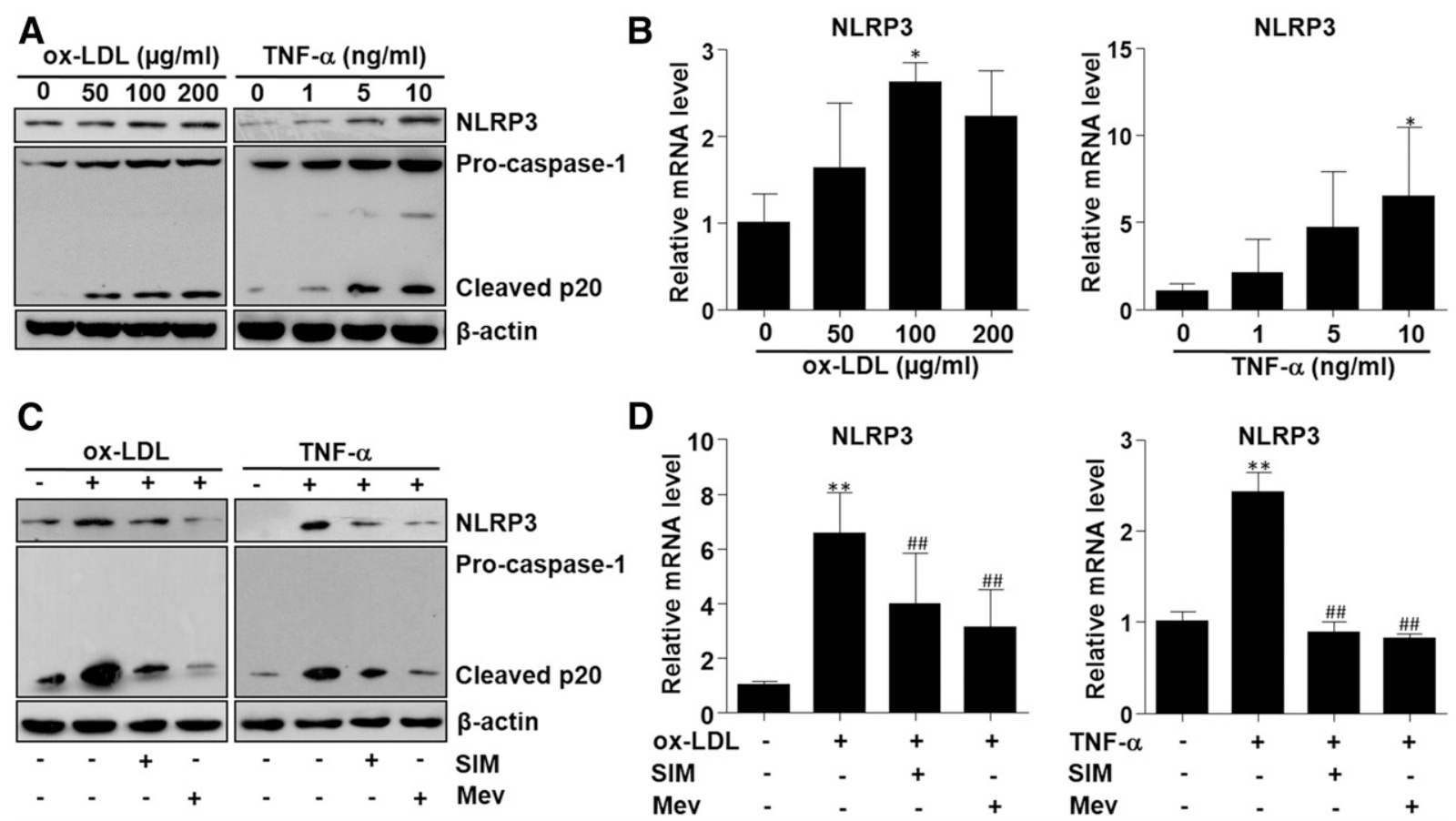

Fig. 1. Effects of statins on ox-LDL- or TNF $\alpha$-induced NLRP3 inflammasome activation in ECs. (A) HUVECs were treated with ox-LDL (0-200 $\mu$ g/ml) or TNF $\alpha(0-10 \mathrm{ng} / \mathrm{ml})$ for 12 hours. Cell lysates were analyzed for NLRP3 and caspase-1 proteins by Western blotting. (B) Total RNA was extracted and subjected to qRT-PCR for the assessment of NLRP3 mRNA. (C) HUVECs were pretreated with simvastatin (SIM; $0.5 \mu \mathrm{M}$ ) or mevastatin (Mev; $5 \mu \mathrm{M}$ ) for 12 hours before stimulation with ox-LDL $(100 \mu \mathrm{g} / \mathrm{ml})$ or TNF $\alpha(10 \mathrm{ng} / \mathrm{ml})$ for 12 hours. Cell lysates were analyzed for NLRP3 and caspase-1 protein levels by Western blotting. (D) Total RNA was extracted and subjected to qRT-PCR for the assessment of NLRP3 mRNA. Data represent the mean \pm SD, $n=3-5$, $* P<0.05, * * P<0.01$ versus control; \#\#P<0.01 versus ox-LDL. 

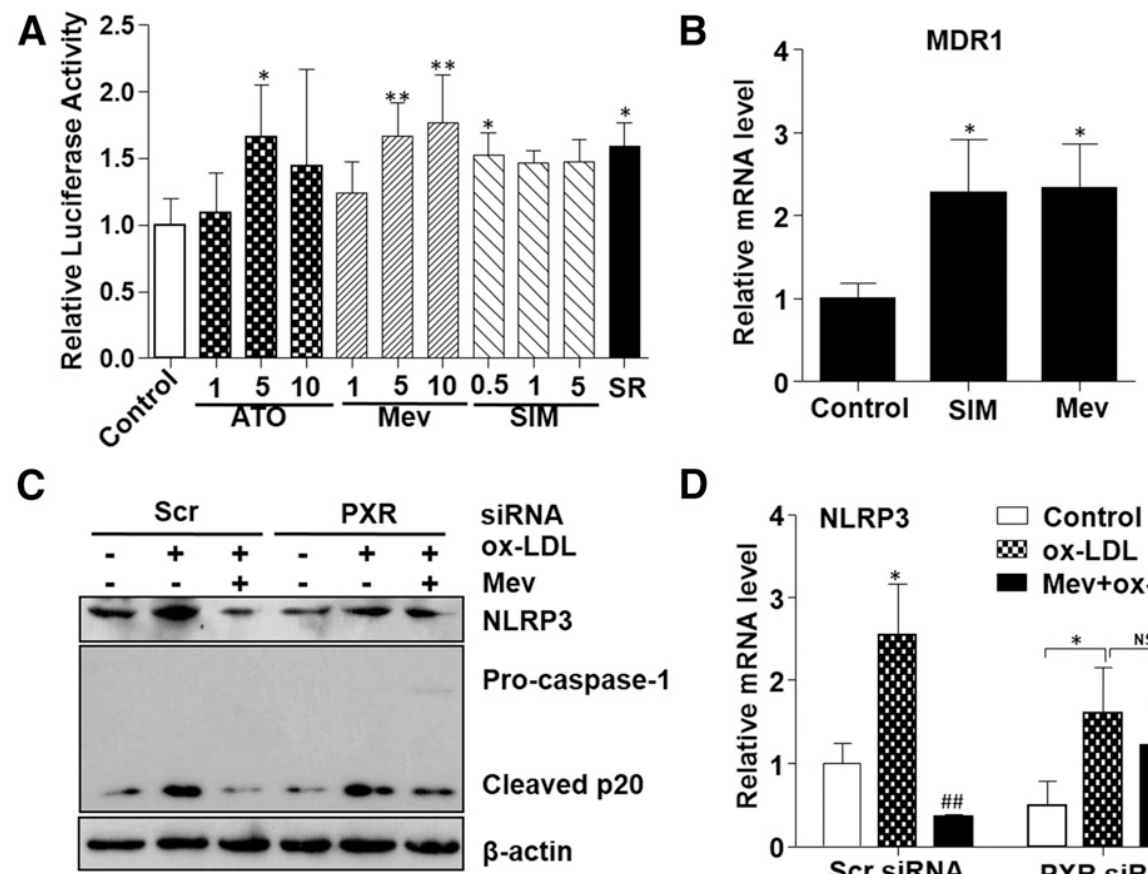

D

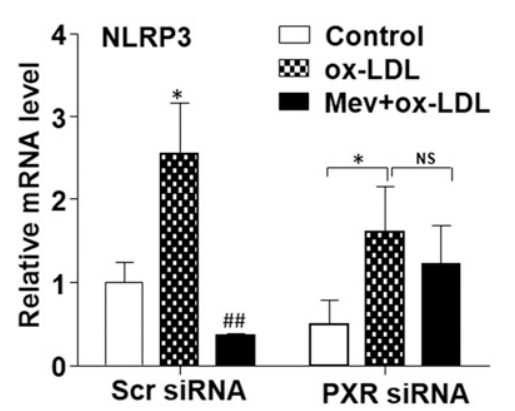

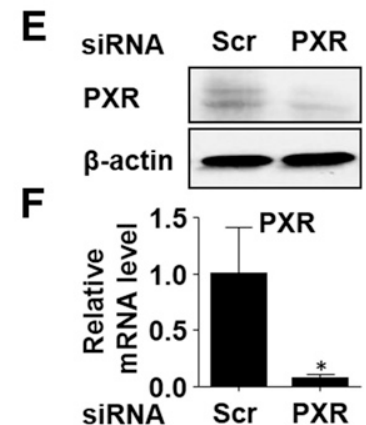

Fig. 2. Effect of PXR knockdown on the effects of statins on ox-LDL- or TNF $\alpha$-activated NLRP3 inflammasome in ECs. (A) BAECs were transfected with PXRE-Luc and pCMX-PXR and, 24 hours later, exposed to atorvastatin (ATO; $1-10 \mu \mathrm{M}$ ), mevastatin (Mev; $1-10 \mu \mathrm{M}$ ), or simvastatin (SIM; 0.5-5 $\mu \mathrm{M}$ ) for 24 hours. Luc activities were measured and normalized to $\beta$-galactosidase activity. SR12813 $(1 \mu \mathrm{M})$ was used as a positive control. (B) HUVECs were incubated with SIM $(0.5 \mu \mathrm{M})$ or $\mathrm{Mev}(5 \mu \mathrm{M})$ for 12 hours. The mRNA level of MDR1 was measured by using qRT-PCR. (C) HUVECs were transfected with PXR siRNA or scrambled (Scr) siRNA for 24 hours and then pretreated with Mev for 12 hours before exposure to ox-LDL (100 $\mu \mathrm{g} / \mathrm{ml}, 12 \mathrm{hours}$ ). (D) qRTPCR for the assessment of NLRP3. (E) PXR protein was detected by Western blotting. (F) The relative mRNA level of PXR was assessed by qRT-PCR. Data represent the mean $\pm \mathrm{SD}, n=3,{ }^{*} P<0.05,{ }^{*} P<0.01$ versus control; \#\#P<0.01 versus ox-LDL; NS, not significant.

from Cell Signaling Technology (Danvers, MA). Antibodies against $\mathrm{PXR}, \mathrm{I} \kappa \mathrm{B} \alpha$, IgG, glyceraldehyde-3-phosphate dehydrogenase, histone, and $\beta$-actin were from Santa Cruz Biotechnology (Santa Cruz, CA). Antibody against lectin-like ox-LDL receptor (LOX-1) was from Sigma-Aldrich (St. Louis, MO). Human recombinant TNF $\alpha$ was from R\&D Systems (Minneapolis, MN). Reactive oxygen species (ROS) inhibitors (NAC and DPI) were from Sigma-Aldrich. Oxidized lowdensity lipoprotein (ox-LDL) was from Anhui Yiyuan Biotechnology Co., Ltd. (Guangzhou, People's Republic of China). Other reagents were from Sigma-Aldrich.

Quantitative Reverse Transcriptase Polymerase Chain Reaction. Total RNA was isolated using TRIzol (Invitrogen, Carlsbad, CA), then reverse transcribed into cDNA by using the iScript cDNA Synthesis Kit (BIO-RAD, Hercules, CA). Quantitative reverse-transcription polymerase chain reaction (qRT-PCR) was performed by using SYBR Green Supermixes (BIO-RAD) and a 7500 Real-time PCR Machine (Applied Biosystems, Foster City, CA). Fold changes of gene expression were calculated using the $2^{-\Delta \Delta \mathrm{Ct}}$ method. The following qRT-PCR primers were used: NLRP3 forward primer, 5' -CTTCTCTGATGAGGCCCAAG-3', reverse primer, 5'-GCAGCAAACTGGAAAGGAAG-3'; LOX-1 forward primer, 5'-TTACTCTCCATGGTGGTGCC-3' , reverse primer, 5' -AGCTTCTTCTGCTTGTTGCC-3'; MDR1 forward primer, $5^{\prime}$-CTTGTTAGACAGCCTCATA-3', reverse primer, 5'-TCATACAGTCAGAGTTCAC-3'; and glyceraldehyde-3-phosphate dehydrogenase (GAPDH) forward primer, 5'-ACCACAGTCCATGCCATCAC-3', reverse primer, 5' -TCCACCACCCTGTTGCTGTA-3'. Glyceraldehyde-3-phosphate dehydrogenase was used as an internal control.

Western Blotting. Protein samples were extracted with lysis buffer (10 mM Tris- $\mathrm{HCl}$ at $\mathrm{pH} 7.4,150 \mathrm{mM} \mathrm{NaCl}$, and $0.1 \%$ Triton $\mathrm{X}-100)$ supplemented with a protease inhibitor cocktail (Roche Diagnostics, Indianapolis, IN). Cytoplasmic proteins were extracted with hypotonic lysis buffer (10 mM Tris-HCl, pH 7.5, $1.5 \mathrm{mM} \mathrm{MgCl}_{2}$,
$10 \mathrm{mM} \mathrm{KCl}$, and $0.5 \% \mathrm{NP} 40$ ). Nuclear proteins were extracted with high-salt buffer (20 mM Tris-HCl, $1.5 \mathrm{mM} \mathrm{MgCl}_{2}, 420 \mathrm{mM} \mathrm{NaCl}, 10 \%$ glycerol, and $0.2 \mathrm{mM}$ EGTA). BCA protein assay reagents (Pierce, Rockford, IL) were used to assess protein concentration. Protein samples were separated on SDS-PAGE and transferred onto a polyvinylidene difluoride membrane. Immunoblotting was performed with primary antibodies and appropriate horseradish peroxidase-conjugated secondary antibodies, visualized using an enhanced chemiluminescence system.

Adenoviral Vectors and Infection. Recombinant adenoviruses (Ads) encoding VP-PXR (Ad-VP-PXR), Ad-tTA [an Ad expressing tetracycline-responsive transactivator (tTA)], Ad-LacZ ( $\beta$-galactosidase), and $\mathrm{Ad}-\mathrm{I} \kappa \mathrm{B} \alpha$ were as previously described (Wang et al., 2013). HUVECs were coinfected with Ad-tTA and Ad-VP-PXR in the presence or absence of tetracycline $(0.1 \mu \mathrm{g} / \mathrm{ml})$.

Small interfering RNA and Transfection. HUVECs were transfected with PXR small interfering RNA (siRNA) (sense, 5'-CAGGAGCAAUUCGCCAUUATT-3'; antisense, 5'-UAAUGGCGAAUUGCUCCUGTT-3'), LOX-1 siRNA (sense, 5'-GGUCUUCAGUUUCUUUACUTT-3'; antisense, 5'-AGUAAAGAAACUGAAGACCTT-3'), or scrambled siRNA (sense, 5'-CGGGUUGCCCAAAGACGACAA-3'; antisense, 5'-UUGUCGUCUUUGGGCAACCCG-3') with Lipofectamine 2000 (Invitrogen). Experiments were performed with these cells at 24 hours after transfection.

Chromatin Immunoprecipitation Assay. HUVECs were treated with $1 \%$ formaldehyde for DNA-protein crosslinking, and then chromatin was sheared by sonication (three times $\times 15$ seconds). Immunoprecipitations were performed with the use of a rabbit polyclonal antibody against NF- $\kappa \mathrm{B} / \mathrm{p} 65$ or control IgG and protein $\mathrm{A} / \mathrm{G}$ Sepharose beads. After washing, the beads were eluted in elution buffer ( $1 \%$ SDS, $100 \mathrm{mM} \mathrm{NaHCO}_{3}$ ). DNA samples were extracted and purified after proteinase $\mathrm{K}$ digestion. The bound DNAs were amplified using qPCR with primers flanking the NF- $\kappa$ B binding sites in the human NLRP3 promoter regions (Table 1). Relative DNA binding was 

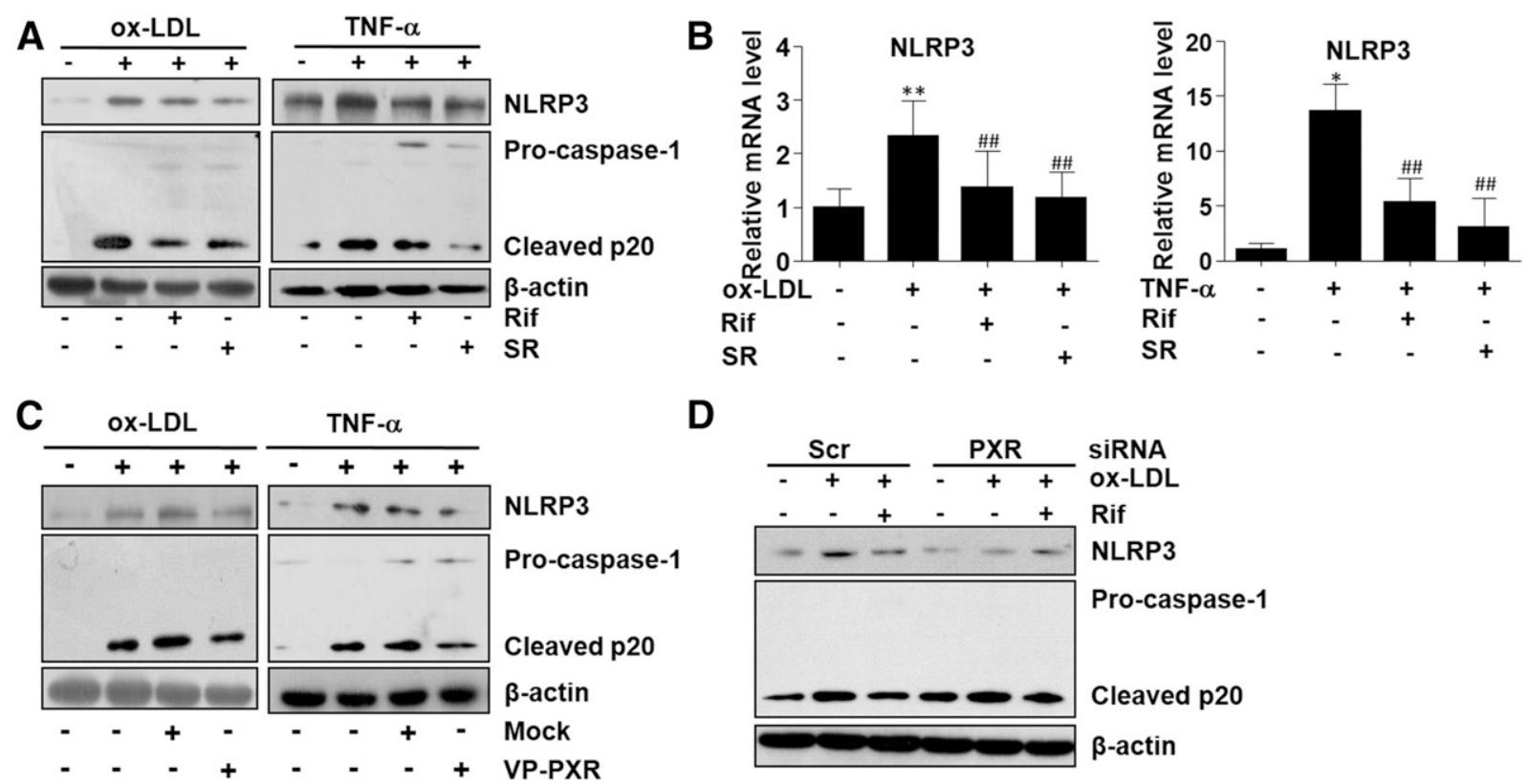

D
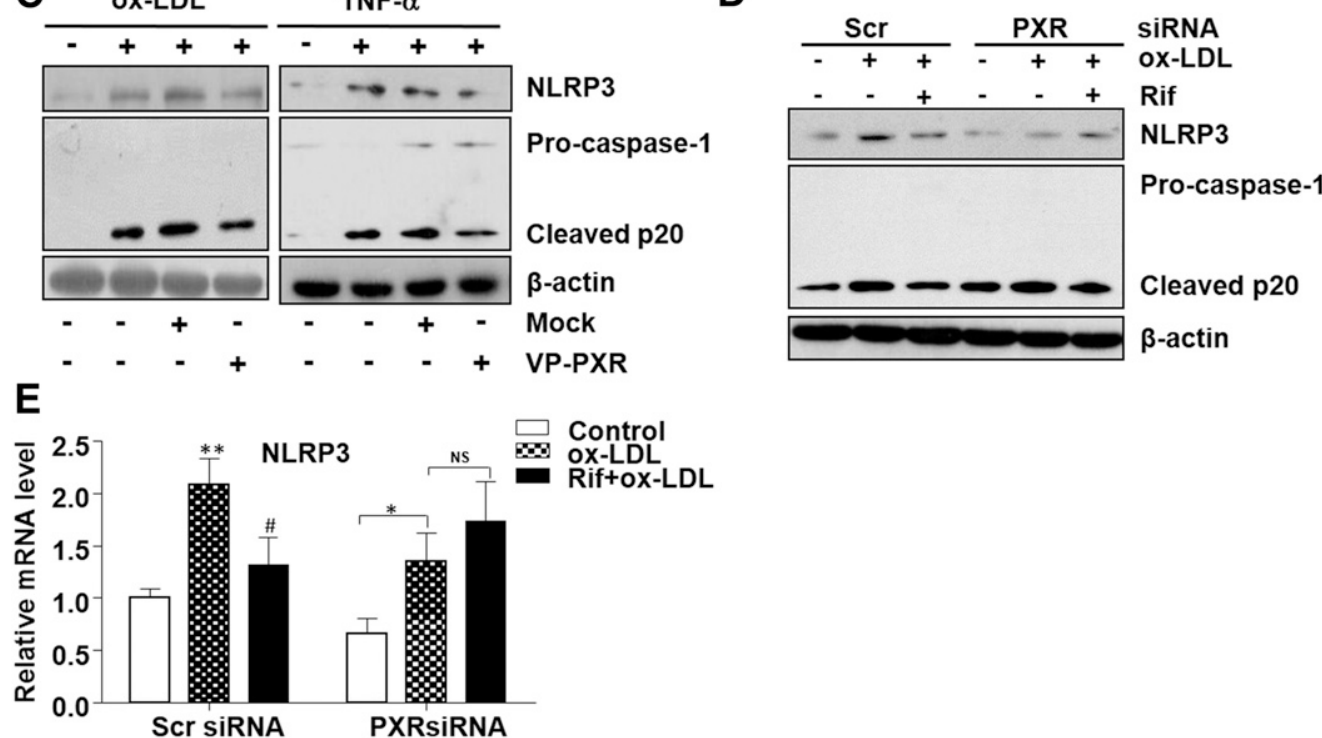

Fig. 3. Effects of PXR agonists on ox-LDL- and TNF $\alpha$-induced NLRP3 inflammasome activation. (A) HUVECs were pretreated with rifampicin (Rif; $10 \mu \mathrm{M})$ or SR12813 (SR; $1 \mu \mathrm{M}$ ) for 12 hours and then incubated with ox-LDL (100 $\mu \mathrm{g} / \mathrm{ml})$ or TNF $\alpha$ (10 ng/ml) for 12 hours. Protein levels of NLRP3 and caspase-1 were assessed by Western blotting. (B) The NLRP3 mRNA level was assessed using qRT-PCR. (C) HUVECs were infected with Ad-VP-PXR for 24 hours and then incubated with ox-LDL or TNF $\alpha$ for 12 hours. NLRP3 and caspase-1 protein levels were assessed by Western blotting. (D) HUVECs were transfected with PXR or scrambled (Scr) siRNA for 24 hours and then pretreated with Rif for 12 hours before exposure to ox-LDL. The protein levels of NLRP3 and caspase-1 were measured by Western blotting. (E) NLRP3 mRNA level was assessed by qRT-PCR. Data are expressed as the mean \pm SD, $n=3-4,{ }^{*} P<0.05, * * P<0.01$ versus control; $\# P<0.05$, \#\#P<0.01 versus ox-LDL or TNF $\alpha$; NS, not significant.

expressed as fold enrichment compared with immunoprecipitates using control IgG (Fan et al., 2008).

Plasmids and Transfection. The genomic fragment harboring -2977 to +151 base pairs in relation to the transcription start site (Anderson et al., 2008) of the human NLRP3 gene was cloned using PCR from the genomic DNA. The primer sequences were 5' -CGGGCTAGCGGTCATACGGTAGTTCTA-3' (forward) and $5^{\prime}$-CGGCTCGAGGCCAGAAGAAATTCCTAG-3' (reverse). The amplified product was subcloned into a pGL3-basic plasmid containing the firefly luciferase (Luc) reporter gene (Promega, Madison, WI) with the use of NheI and XhoI restriction enzymes to generate NLRP3-Luc. The PXR-responsive element (PXRE)-Luc reporter plasmid pCMX-PXR and $5 \times$ NF- $\kappa$ B-Luc have been described previously (Wang et al., 2013). Plasmids were transfected into BAECs using Lipofectamine 2000. To normalize transfection efficiency, a plasmid expressing $\beta$-galactosidase (pRSV-gal) was cotransfected. Results were expressed as fold induction compared with basal promoter activity.

Statistical Analysis. Data are presented as the mean \pm SD from at least three independent experiments. Data analysis was performed using Microsoft Excel (Microsoft, Redmond, WA) and GraphPad Prism (version 6; GraphPad Software, La Jolla, CA). Student's $t$ test (paired groups) or one-way analysis of variance followed by Newman-Keuls post hoc test (multigroup comparisons) were used to analyze the statistical significance. A $P$ value of $<0.05$ was considered to be significant.

\section{Results}

Effects of Statins on ox-LDL- or TNF $\alpha$-induced NLRP3 Inflammasome Activation in ECs. Ox-LDL and $\mathrm{TNF} \alpha$ are critical atherogenic mediators known to induce the expression of proinflammatory genes in ECs (Yokode, 2001; Zhang et al., 2009). To test the effects of ox-LDL and TNF $\alpha$ on the NLRP3 inflammasome in ECs, we treated HUVECs with various concentrations of ox-LDL (0 to $\sim 200 \mu \mathrm{g} / \mathrm{ml})$ or TNF $\alpha$ (0 to $\sim 10 \mathrm{ng} / \mathrm{ml}$ ) for 12 hours. Western blotting showed that both ox-LDL and TNF $\alpha$ dose-dependently increased the protein levels of NLRP3 and cleaved caspase-1 (Fig. 1A). A similar dose-dependent action was observed at the NLRP3 mRNA level as measured by using qRT-PCR (Fig. 1B). Next, we examined the effects of statins on NLRP3 inflammasome activation. We treated HUVECs with simvastatin $(0.5 \mu \mathrm{M})$ or mevastatin $(5 \mu \mathrm{M})$ for 12 hours before exposure to ox-LDL or $\mathrm{TNF} \alpha$. As shown in Fig. 1C, simvastatin or mevastatin significantly inhibited the ox-LDL- or TNF $\alpha$-induced increases in protein levels of NLRP3 and cleaved caspase-1. The mRNA level of NLRP3 was also decreased by the statins (Fig. 1D). Taken together, these results indicated that statins attenuate the activation of NLRP3 inflammasome induced by ox-LDL or $\mathrm{TNF} \alpha$ in ECs. 

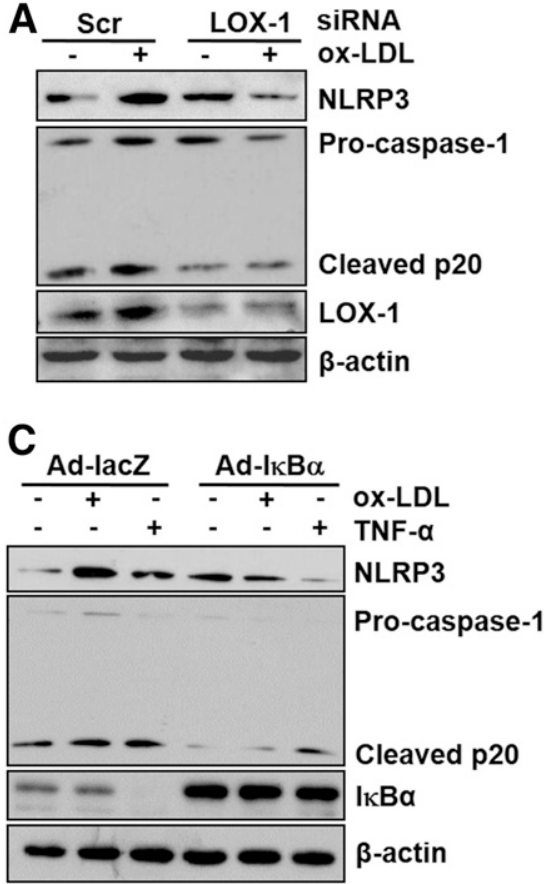

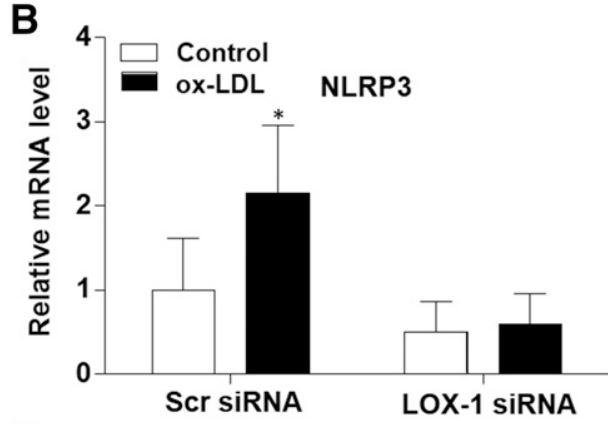

D

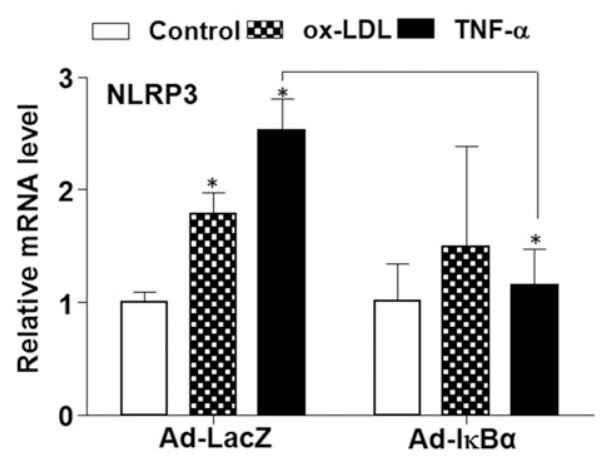

Fig. 4. Effects of LOX-1 and NF- $\kappa$ B knockdown on activation of NLRP3 inflammasome by ox-LDL or TNF $\alpha$. (A) HUVECs were transfected with LOX-1 or scrambled (Scr) siRNAs for 24 hours and then incubated with ox-LDL for 12 hours. Cell lysates were analyzed for NLRP3 and caspase-1 protein levels by Western blotting. (B) Total RNA was extracted and subjected to qRT-PCR for the assessment of NLRP3 mRNA. (C) HUVECs were infected with Ad-Lac or Ad-I $\kappa \mathrm{B} \alpha$ for 24 hours and then simulated with ox-LDL or $\mathrm{TNF} \alpha$ for 12 hours. Protein levels of NLRP3 and caspase-1 were assessed by Western blotting. (D) NLRP3 mRNA level was assessed by qRT-PCR. Data represent the mean $\pm \mathrm{SD}, n=3-4, * P<0.05$ versus control.
Role of PXR in the Suppressive Effect of Statins on ox-LDL- or TNF $\alpha$-Activated NLRP3 Inflammasome in ECs. Statins have been reported to activate PXR in liver cells (Howe et al., 2011; Hoffart et al., 2012; Plée-Gautier et al., 2012). Thus, we examined the effect of statins on PXR in ECs. We cotransfected pCMX-PXR and PXRE-Luc into BAECs and then treated with atorvastatin, mevastatin, or simvastatin at various concentrations for 24 hours. PXR reporter activity was significantly increased by all three statins (Fig. 2A). In HUVECs, simvastatin and mevastatin also increased the mRNA level of MDR1, a PXR target gene (Fig. 2B). These results indicated that statins can activate PXR in ECs.

To determine whether PXR mediates the inhibitory effect of statins on inflammasome activation, we used siRNA to silence the expression of endogenous PXR in ECs. The suppressive effects of the statins on the ox-LDL-activated NLRP3 inflammasome were significantly reduced in ECs transfected with PXR siRNA (Fig. 2, C and D). Knockdown of PXR protein and mRNA levels were validated with Western blotting and qRTPCR (Fig. 2, E and F). Together, these results demonstrated that statins attenuated ox-LDL- or TNF $\alpha$-activated NLRP3 inflammasome activation via a PXR-dependent mechanism.

Effects of PXR Agonists on ox-LDL- or TNF $\alpha$-Induced NLRP3 Inflammasome. To examine whether other specific PXR agonists have a similar inhibitory effect on NLRP3 inflammasome activation, we pretreated ECs with rifampicin $(10 \mu \mathrm{M})$ or SR12813 $(1 \mu \mathrm{M})$ before exposure to ox-LDL or $\mathrm{TNF} \alpha$. As shown in Fig. 3A, rifampicin and SR12813 both inhibited the ox-LDL- or TNF $\alpha$-stimulated increases in NLRP3 and cleaved caspase-1. The mRNA level of NLRP3 was also decreased (Fig. 3B). To rule out a potential off-target effect of individual agonists, we infected HUVECs with Ad-VP-PXR. We found that overexpression of PXR attenuated the NLRP3 inflammasome activation in response to ox-LDL or TNF $\alpha$ (Fig. 3C). Furthermore, PXR knockdown abrogated the suppressive effect of rifampicin on ox-LDL-induced NLRP3 inflammasome activation (Fig. 3, D and E). Taken together, these results suggested that PXR agonists inhibit ox-LDL- or TNF $\alpha$ activated NLRP3 inflammasome in a PXR-dependent manner in ECs.

Role of the LOX-1/NF- B Pathway in Activation of the NLRP3 Inflammasome by ox-LDL or TNF $\alpha$. LOX-1 is the major receptor for ox-LDL in ECs and is thought to be a marker of endothelial dysfunction in many cardiovascular diseases (Pirillo et al., 2013; Ulrich-Merzenich and Zeitler, 2013). LOX-1 expression was increased after ox-LDL or TNF $\alpha$ exposure (Supplemental Fig. 1). Knockdown of LOX-1 in ECs attenuated the activation of NLRP3 inflammasome induced by ox-LDL (Fig. 4, A and B). These results indicated an important role of LOX-1 in ox-LDL activation of the NLRP3 inflammasome in ECs. It has been previously shown (Hu et al., 2008) that ox-LDL binding to endothelial LOX-1 can activate the $\mathrm{NF}-\kappa \mathrm{B}$ signal pathways. We investigated whether $\mathrm{NF}-\kappa \mathrm{B}$ was also involved in ox-LDL- or TNF $\alpha$-induced NLRP3 inflammasome activation. HUVECs were infected with $\mathrm{Ad}-\mathrm{I} \kappa \mathrm{B} \alpha$ to block NF- $\kappa$ B activation before exposure to ox-LDL or TNF $\alpha$. As shown in Fig. 4, $\mathrm{C}$ and $\mathrm{D}, \mathrm{Ad}-\mathrm{I} \kappa \mathrm{B} \alpha$ abolished both ox-LDLand $\mathrm{TNF} \alpha$-induced NLRP3 inflammasome activation. These results indicated that the LOX-1/NF- $\kappa$ B pathway contributes to the activation of the NLRP3 inflammasome by ox-LDL or $\mathrm{TNF} \alpha$.

Role of the LOX-1/NF- $\kappa$ B Pathway in the Effects of Statins on the NLRP3 Inflammasome. To investigate whether statins attenuate NLRP3 inflammasome activation by inhibiting the LOX-1/NF- $\kappa$ B pathway, we pretreated ECs with statins for 12 hours before stimulation with ox-LDL. As shown in Fig. 5, A and B, ox-LDL increased LOX-1 expression; this response was significantly diminished by simvastatin or mevastatin. Similarly, PXR agonists (rifampicin and SR12813) or the overexpression of PXR also decreased protein 


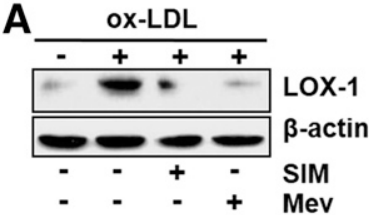

B

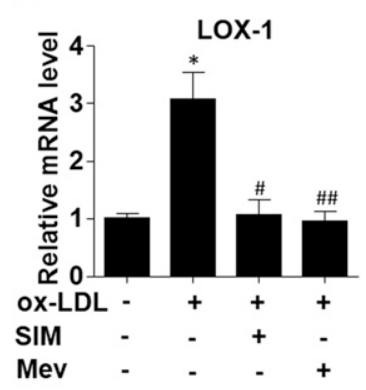

C

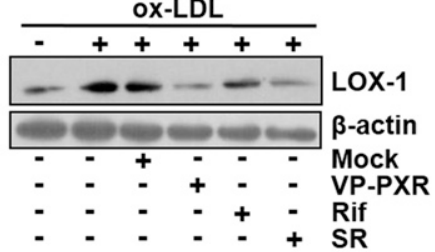

D

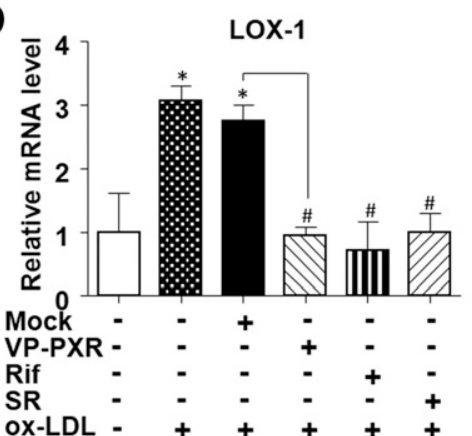

E

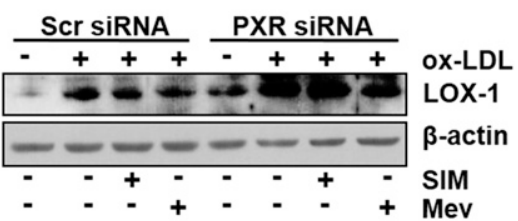

$\mathbf{F}$

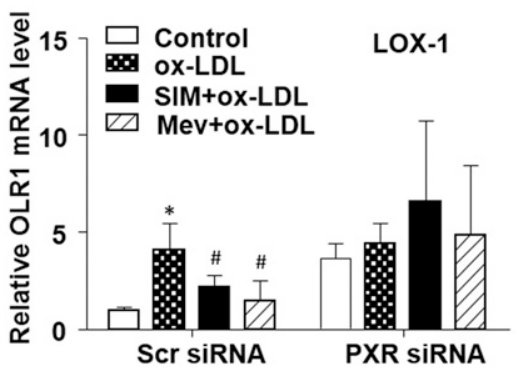

G
H

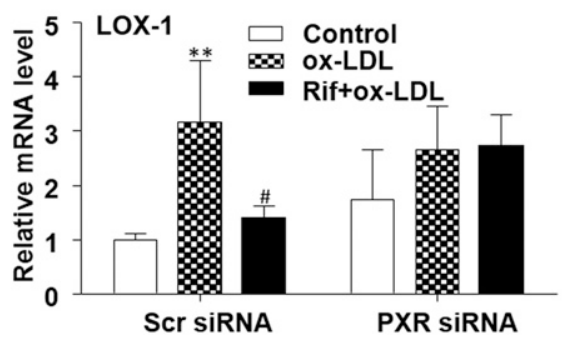

Fig. 5. Effects of statins on NLRP3 inflammasome with LOX-1/NF- $\kappa$ B knockdown. (A) HUVECs were treated with simvastatin (SIM; $0.5 \mu M$ ) and mevastatin (Mev; $5 \mu \mathrm{M}$ ) for 12 hours before exposure to ox-LDL for 12 hours. Protein level of LOX-1 was assessed by Western blotting. (B) LOX-1 mRNA level was assessed by qRT-PCR. (C) HUVECs were infected with VP-PXR (24 hours), rifampicin (Rif) (12 hours), or SR12813 (SR) (12 hours) before exposure to ox-LDL. LOX-1 protein was assessed by Western blotting. (D) LOX-1 mRNA was assessed by qRT-PCR. (E) HUVECs were transfected with PXR or scrambled (Scr) siRNA for 24 hours, and then pretreated with SIM or Mev for 12 hours before exposure to ox-LDL for 12 hours. LOX-1 protein was assessed by Western blotting. (F) LOX-1 mRNA was assessed by qRT-PCR. (G) HUVECs were transfected with PXR or Scr siRNA for 24 hours and then pretreated with Rif for 12 hours before exposure to ox-LDL. LOX-1 protein levels were assessed. (H) LOX-1 mRNA level was measured by qRT-PCR. Data represent the mean $\pm \mathrm{SD}, n=3-4, * P<0.05,{ }^{* *} P<0.01$ versus control; $\# P<0.05$, \#\#P<0.01 versus ox-LDL.

and mRNA levels of LOX-1 (Fig. 5, C and D). Furthermore, PXR knockdown abolished the suppressive effects of statins and rifampicin on LOX-1 expression (Fig. 5, E-H). Taken together, these results indicated an inhibitory effect of PXR on LOX-1 expression.

LOX-1 activation results in the excessive generation of ROSs, which are known to trigger the activation of the NLRP3 inflammasome (Schroder and Tschopp, 2010). We found that simvastatin or mevastatin significantly reduced ROS generation in response to $\mathrm{TNF} \alpha$ (Supplemental Fig. 2). Furthermore, the PXR agonists rifampicin and SR12813 also decreased NF- $\kappa$ B activation by ox-LDL or TNF $\alpha$ (Supplemental Fig. 3). Thus, these results suggested that PXR inhibition of ROSs may contribute to the inhibitory effects of statins on activation of the LOX-1/NF- $\kappa$ B pathway and the NLRP3 inflammasome.

Effects of Statins on PXR Inhibition of NF- $\boldsymbol{B}$ Binding to the NLRP3 Promoter. Since NF- $\kappa$ B plays a central role in the transcriptional regulation of the NLRP3 gene and PXR suppressed activation of NF- $\kappa$ B target genes (Gu et al., 2006; Xie and Tian, 2006; Qiao et al., 2012), we examined the effects of statin-activated PXR on NF- $\kappa$ B binding activity to the human NLRP3 promoter. Bioinformatics analysis using the Transcription Element Search System (http:// www.cbil.upenn.edu/cgi-bin/tess/te) revealed three canonical
$\mathrm{NF}-\kappa \mathrm{B}-$ responsive elements in the $5^{\prime}$-flanking region of the human NLRP3 gene (Fig. 6A). Chromatin immunoprecipitation (ChIP) assays demonstrated that both ox-LDL and $\mathrm{TNF} \alpha$ increased NF- $\mathrm{B}$ binding at these motifs and that these responses were inhibited by mevastatin (Fig. 6B) or SR12813 (Fig. 6C). Furthermore, we performed the NLRP3 promoter reporter assay in BAECs. As shown in Fig. 6D, $\mathrm{TNF} \alpha$ increased the NLRP3-Luc reporter activity, which was inhibited by SR12813. These results indicated that the statinactivated PXR inhibits NF- $\kappa \mathrm{B}$ binding to the NLRP3 promoter in ECs.

\section{Discussion}

In this study, we demonstrated for the first time that statins can inhibit ox-LDL- or TNF $\alpha$-induced NLRP3 inflammasome activation in ECs and that this inhibitory effect is mediated by activation of the xenobiotic nuclear receptor PXR.

A variety of intracellular and extracellular stimuli have been known to trigger the activation of NLRP3 inflammasomes. Such "danger" signals include microbial components, ATP, glucose, cholesterol crystal, hyaluronan, and ROS (Jin and Flavell, 2010; Sutterwala et al., 2014). As part of an innate immunity system, the NLRP3 inflammasome is essential to the maintenance of homeostasis against pathogens and 
A

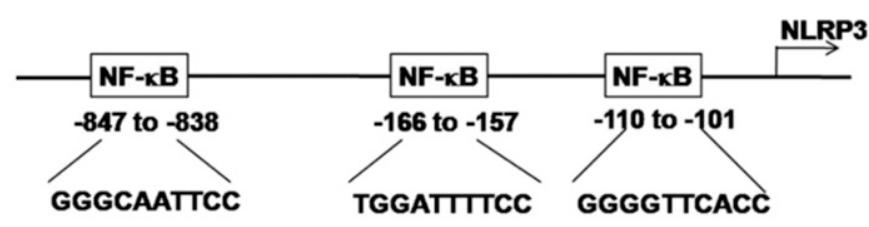

B

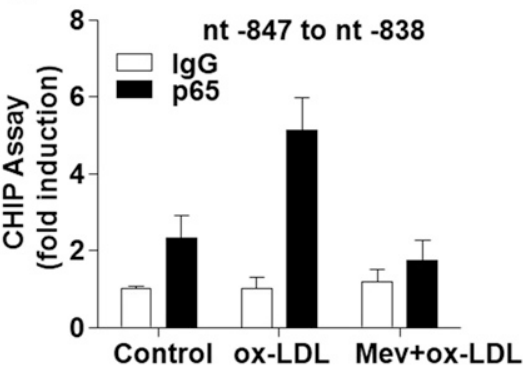

C

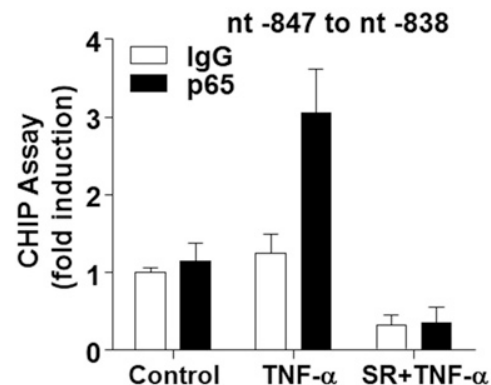

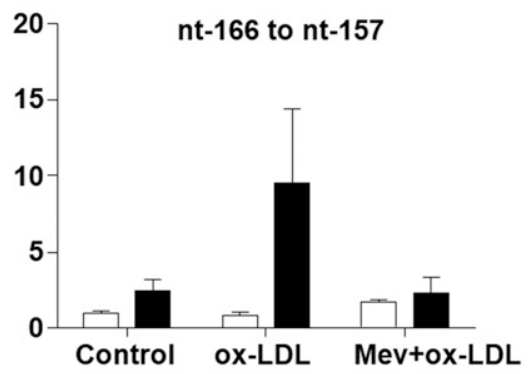

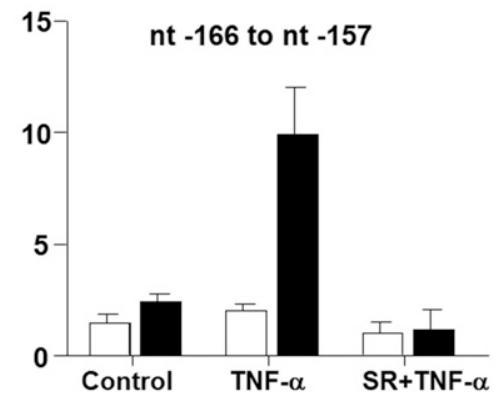

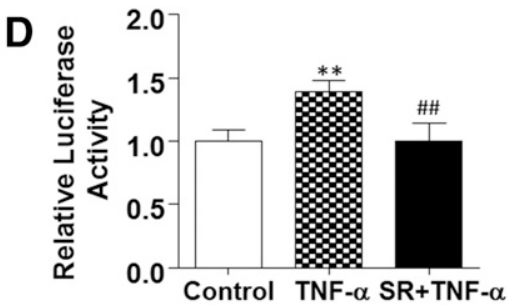
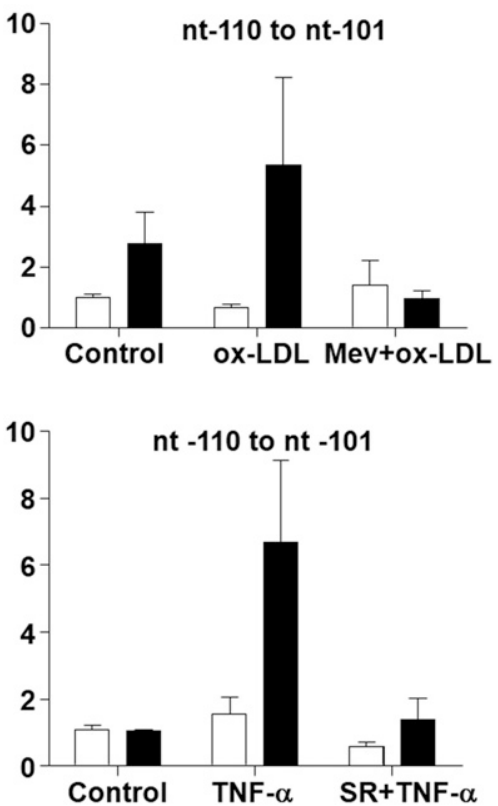

Fig. 6. Effects of statins and PXR on NF- $\kappa$ B binding to the NLRP3 promoter. (A) The diagram depicts putative NF- $\kappa$ B motifs in the $5{ }^{\prime}$-flanking region of the human NLRP3 gene. HUVECs were treated with ox-LDL (B) or TNF $\alpha$ (C) in the presence or absence of mevastatin (Mev) or simvastatin (SR). ChIP assays were performed with NF- $\mathrm{B} / \mathrm{p} 65$ antibody or IgG as a negative control. Immunoprecipitated DNA was eluted and amplified by PCR using specific primers flanking the NF- $\kappa$ B sites. PCR results were expressed as fold change compared with IgG control. (D) BAECs were cotransfected with NLRP3-Luc plasmid then were exposed to SR for 12 hours before incubating with TNF $\alpha$. Luc activity was measured and normalized to $\beta$-gal activity. Data represent the mean $\pm \mathrm{SD}, n=4, * * P<0.01$ versus control; \#\#P<0.01 versus $\mathrm{TNF} \alpha$.

xenobiotics. However, excessive activation of inflammasomes may lead to tissue damage and is implicated in proinflammatory diseases (Kim and Jo, 2013). Cholesterol crystals and ox-LDL activate NLRP3 inflammasome in macrophages and play pathologic roles in atherosclerosis (Duewell et al., 2010; Sheedy et al., 2013). A randomized clinical study (Satoh et al., 2014) has shown that mRNA and protein levels of NLRP3 in peripheral blood mononuclear cells are higher in the CAD patients and are positively correlated with the plasma levels of IL- $1 \beta$ and IL-18. Interestingly, NLRP3 and IL- $1 \beta / \mathrm{IL}-18$ levels were significantly reduced after treatment with atorvastatin for 8 months. In the current study, we found that ox-LDL and $\mathrm{TNF} \alpha$ triggered NLRP3 inflammasome activation in ECs and, most importantly, that statins inhibited ox-LDL- or TNFtriggered NLRP3 inflammasome activation (Fig. 1).

One of the important findings in this study is that PXR plays a critical role in the suppression of $\mathrm{TNF} \alpha$ - and ox-LDLinduced NLRP3 inflammasome activation in human ECs. This notion is supported by the following results: 1) statins (simvastatin, mevastatin, and atorvastatin) activated the PXR reporter and endogenous target gene MDR1 in ECs (Fig. 2, A and B); and 2) gene silencing of PXR abrogated the suppressive effect of statin on NLRP3 inflammasome (Fig. 2, C and D), whereas PXR agonists or overexpression of PXR elicited similar effects (Fig. 3, A-C). It is worth noting that the pharmacologic actions of PXR are highly divergent across species (Zhou et al., 2009). The species divergence may be explained by the low homology in the ligand-binding domain, where human and mouse genes share less than $80 \%$ in amino acid sequences (Zhou et al., 2009). Thus, we used rifampicin and SR12813, two potent agonists for human PXR with little agonism toward rodent PXR (Jones et al., 2000).

Atherogenic effects of LOX-1 have been well established and are involved in endothelial dysfunction, foam cell formation, platelet activation, and plaque instability (Pirillo et al., 2013). Expression of LOX-1 can be induced in response to many atherogenic stimuli, including $\mathrm{TNF} \alpha$, angiotensin II, endothelin-1, free radicals, and ox-LDL (Pirillo et al., 2013). LOX-1 activation leads to ROS generation, mitochondrial DNA damage, and the induction of NLRP3 expression in THP-1 cells (Ding et al., 2014). We found that LOX-1 was essential for ox-LDL-triggered NLRP3 inflammasome activation in ECs (Fig. 4, A and B). Activation of LOX-1 by ox-LDL triggers the NF- $\kappa \mathrm{B}$ signaling pathway (Matsunaga et al., 2003; Mattaliano et al., 2009). Here, we demonstrated that the PXR activation by treatment with either statins or rifampicin, or by PXR overexpression, inhibited LOX-1 expression (Fig. 5, A-D). Conversely, knockdown of PXR diminished the inhibitory effects of statins and rifampicin on LOX-1 (Fig. 5, E-H), indicating that statins inhibit LOX-1 
expression in a PXR-dependent manner. These results suggest that inhibition of LOX-1/NF- $\kappa \mathrm{B}$ is a key mechanism by which statin/PXR signaling inhibits ox-LDL- or TNF $\alpha$ - induced NLRP3 inflammasome activation in ECs (Fig. 4, C and D). This finding is consistent with the previously described reciprocal inhibition between $\mathrm{PXR}$ and $\mathrm{NF}-\kappa \mathrm{B}$ pathways (Zhou et al., 2006). In light of the well-established roles of NF- $\kappa \mathrm{B}$ in atherogenesis (Baker et al., 2011) and the pleotropic effects of statins on endothelial proinflammatory response (Greenwood and Mason, 2007), the PXR/NF$\kappa \mathrm{B} / \mathrm{NLRP} 3$-inflammasome signaling pathway may represent a novel mechanism underlying the atheroprotective actions of statins.

Intriguingly, our previous study (Wang et al., 2014) showed that PXR has recurrent cis-elements in the regulatory regions of NLRP3 genes that, when activated, can transcriptionally induce the expression of NLRP3. Such a mechanism may play an important role in the maintenance of vascular homeostasis by coupling endothelial detoxification with immune surveillance program against the xenobiotics and concurrent tissue injury. On the other hand, atherogenic stimuli provoke excessive expression of NLRP3 and a much more robust activation of the NLRP3 inflammasome via the activator protein 1 (Yang et al., 2014) and NF- $\kappa$ B pathways. The results in the present study also revealed functional $\mathrm{NF}-\kappa \mathrm{B}$ motifs in the NLRP3 promoter region. Statin or the PXR agonist SR12813 diminished NF- $\kappa$ B/p65 binding to these motifs, supporting the fact that statin-activated PXR can counteract ox-LDL-induced inflammasome activation by blocking the LOX-1/NF- $\kappa$ B pathway. It is also conceivable that the statin-activated PXR may antagonize the NF- $\kappa$ Bmediated transcriptional complexes at the promoter level by "trans-repression," a mechanism involving the competition and relocation of transcriptional cofactors that is responsible for the anti-inflammatory actions of nuclear receptors (Glass, 2006; Gu et al., 2006). Furthermore, our recent study demonstrated that laminar shear stress is an atheroprotective flow pattern that enhances endothelial PXR activity. The shear stress significantly suppresses the proinflammatory response but promotes endothelial survival in response to xenobiotic injuries, reinforcing the importance of the context- and tissue-specific effects of PXR in ECs (Wang et al., 2013).

In conclusion, our results demonstrate that statins inhibit NLRP3 inflammasome via PXR activation in ECs. Since the conversion of endothelium to a proinflammatory state is an early step in atherogenesis, the suppressive role of statins on endothelial inflammasome activation may provide new molecular insights into their pharmacological actions in the primary prevention of cardiovascular morbidity and mortality.

\section{Acknowledgments}

The authors would like to thank Yahan Liu for helpful discussion.

\section{Authorship Contributions}

Participated in research design: Lei, Xiao, and N. Wang.

Conducted experiments: S. Wang, Xie, K. Zhang, Z. Zhang, and Xiao.

Contributed new reagents or analytic tools: Lai.

Performed data analysis: Lai, Mao, Xiao, and N. Wang.

Wrote or contributed to the writing of the manuscript: S. Wang, Guan, Xiao, and N. Wang.

\section{References}

Anderson JP, Mueller JL, Misaghi A, Anderson S, Sivagnanam M, Kolodner RD, and Hoffman HM (2008) Initial description of the human NLRP3 promoter. Genes Immun 9:721-726.

Baker RG, Hayden MS, and Ghosh S (2011) NF-кB, inflammation, and metabolic disease. Cell Metab 13:11-22.

Davignon J and Ganz P (2004) Role of endothelial dysfunction in atherosclerosis. Circulation 109 (Suppl 1):III27-III32.

Ding Z, Liu S, Wang X, Dai Y, Khaidakov M, Deng X, Fan Y, Xiang D, and Mehta JL (2014) LOX-1, mtDNA damage, and NLRP3 inflammasome activation in macrophages: implications in atherogenesis. Cardiovasc Res 103:619-628.

Duewell P, Kono H, Rayner KJ, Sirois CM, Vladimer G, Bauernfeind FG, Abela GS, Franchi L, Nuñez G, Schnurr M, et al. (2010) NLRP3 inflammasomes are required for atherogenesis and activated by cholesterol crystals. Nature 464:1357-1361.

Fan Y, Wang Y, Tang Z, Zhang H, Qin X, Zhu Y, Guan Y, Wang X, Staels B, Chien S, et al. (2008) Suppression of pro-inflammatory adhesion molecules by PPAR-delta in human vascular endothelial cells. Arterioscler Thromb Vasc Biol 28:315-321.

Fearon WF and Fearon DT (2008) Inflammation and cardiovascular disease: role of the interleukin-1 receptor antagonist. Circulation 117:2577-2579.

Glass CK (2006) Going nuclear in metabolic and cardiovascular disease. J Clin Invest 116:556-560.

Greenwood J and Mason JC (2007) Statins and the vascular endothelial inflammatory response. Trends Immunol 28:88-98.

Gu X, Ke S, Liu D, Sheng T, Thomas PE, Rabson AB, Gallo MA, Xie W, and Tian Y (2006) Role of NF-kappaB in regulation of PXR-mediated gene expression: a mechanism for the suppression of cytochrome P-450 3A4 by proinflammatory agents. J Biol Chem 281:17882-17889.

Hoffart E, Ghebreghiorghis L, Nussler AK, Thasler WE, Weiss TS, Schwab M, and Burk O (2012) Effects of atorvastatin metabolites on induction of drugmetabolizing enzymes and membrane transporters through human pregnane $\mathrm{X}$ receptor. $\mathrm{Br} J$ Pharmacol 165:1595-1608.

Howe K, Sanat F, Thumser AE, Coleman T, and Plant N (2011) The statin class of HMG-CoA reductase inhibitors demonstrate differential activation of the nuclear receptors PXR, CAR and FXR, as well as their downstream target genes. Xenobiotica 41:519-529.

Hu C, Dandapat A, Sun L, Chen J, Marwali MR, Romeo F, Sawamura T, and Mehta JL (2008) LOX-1 deletion decreases collagen accumulation in atherosclerotic plaque in low-density lipoprotein receptor knockout mice fed a high-cholesterol diet. Cardiovasc Res 79:287-293.

Jin C and Flavell RA (2010) Molecular mechanism of NLRP3 inflammasome activation. J Clin Immunol 30:628-631.

Jones SA, Moore LB, Shenk JL, Wisely GB, Hamilton GA, McKee DD, Tomkinson NC, LeCluyse EL, Lambert MH, Willson TM, et al. (2000) The pregnane X receptor: a promiscuous xenobiotic receptor that has diverged during evolution. Mol Endocrinol 14:27-39.

Kesavan M, Sarath TS, Kannan K, Suresh S, Gupta P, Vijayakaran K, Sankar P, Kurade NP, Mishra SK, and Sarkar SN (2014) Atorvastatin restores arsenicinduced vascular dysfunction in rats: modulation of nitric oxide signaling and inflammatory mediators. Toxicol Appl Pharmacol 280:107-116.

Kim JJ and Jo EK (2013) NLRP3 inflammasome and host protection against bacterial infection. J Korean Med Sci 28:1415-1423.

Kliewer SA, Goodwin B, and Willson TM (2002) The nuclear pregnane X receptor: a key regulator of xenobiotic metabolism. Endocr Rev 23:687-702.

Luo B, Li B, Wang W, Liu X, Xia Y, Zhang C, Zhang Y, Zhang M, and An F (2014) Rosuvastatin alleviates diabetic cardiomyopathy by inhibiting NLRP3 inflammasome and MAPK pathways in a type 2 diabetes rat model. Cardiovasc Drugs Ther 28:33-43.

Matsunaga T, Hokari S, Koyama I, Harada T, and Komoda T (2003) NF-kappa B activation in endothelial cells treated with oxidized high-density lipoprotein. Biochem Biophys Res Commun 303:313-319.

Mattaliano MD, Huard C, Cao W, Hill AA, Zhong W, Martinez RV, Harnish DC, Paulsen JE, and Shih HH (2009) LOX-1-dependent transcriptional regulation in response to oxidized LDL treatment of human aortic endothelial cells. Am $J$ Physiol Cell Physiol 296:C1329-C1337.

Mitroulis I, Skendros P, and Ritis K (2010) Targeting IL-1beta in disease; the expanding role of NLRP3 inflammasome. Eur J Intern Med 21:157-163.

Morimoto K, Janssen WJ, Fessler MB, McPhillips KA, Borges VM, Bowler RP, Xiao YQ, Kench JA, Henson PM, and Vandivier RW (2006) Lovastatin enhances clearance of apoptotic cells (efferocytosis) with implications for chronic obstructive pulmonary disease. J Immunol 176:7657-7665.

Pirillo A, Norata GD, and Catapano AL (2013) LOX-1, OxLDL, and atherosclerosis. Mediators Inflamm 2013:152786.

Plée-Gautier E, Antoun J, Goulitquer S, Le Jossic-Corcos C, Simon B, Amet Y, Salaün JP, and Corcos L (2012) Statins increase cytochrome P450 4F3-mediated eicosanoids production in human liver cells: a PXR dependent mechanism. Biochem Pharmacol 84:571-579.

Qiao Y, Wang P, Qi J, Zhang L, and Gao C (2012) TLR-induced NF-кB activation regulates NLRP3 expression in murine macrophages. FEBS Lett 586 : 1022-1026.

Satoh M, Tabuchi T, Itoh T, and Nakamura M (2014) NLRP3 inflammasome activation in coronary artery disease: results from prospective and randomized study of treatment with atorvastatin or rosuvastatin. Clin Sci (Lond) 126:233-241.

Schroder K and Tschopp J (2010) The inflammasomes. Cell 140:821-832.

Sheedy FJ, Grebe A, Rayner KJ, Kalantari P, Ramkhelawon B, Carpenter SB Becker CE, Ediriweera HN, Mullick AE, Golenbock DT, et al. (2013) CD36 coordinates NLRP3 inflammasome activation by facilitating intracellular nucleation of soluble ligands into particulate ligands in sterile inflammation. Nat Immunol 14:812-820.

Spyridopoulos I, Haendeler J, Urbich C, Brummendorf TH, Oh H, Schneider MD, Zeiher AM, and Dimmeler S (2004) Statins enhance migratory capacity by 
upregulation of the telomere repeat-binding factor TRF2 in endothelial progenitor cells. Circulation 110:3136-3142.

Strowig T, Henao-Mejia J, Elinav E, and Flavell R (2012) Inflammasomes in health and disease. Nature 481:278-286.

Sutterwala FS, Haasken S, and Cassel SL (2014) Mechanism of NLRP3 inflammasome activation. Ann N Y Acad Sci 1319:82-95.

Ulrich-Merzenich G and Zeitler H (2013) The lectin-like oxidized low-density lipoprotein receptor-1 as therapeutic target for atherosclerosis, inflammatory conditions and longevity. Expert Opin Ther Targets 17:905-919.

Wang S, Lei T, Zhang K, Zhao W, Fang L, Lai B, Han J, Xiao L, and Wang N (2014) Xenobiotic pregnane $\mathrm{X}$ receptor $(\mathrm{PXR})$ regulates innate immunity via activation of NLRP3 inflammasome in vascular endothelial cells. $J$ Biol Chem 289 30075-30081.

Wang X, Fang X, Zhou J, Chen Z, Zhao B, Xiao L, Liu A, Li YS, Shyy JY, Guan Y, et al. (2013) Shear stress activation of nuclear receptor PXR in endothelial detoxification. Proc Natl Acad Sci USA 110:13174-13179.

Xia M, Boini KM, Abais JM, Xu M, Zhang Y, and Li PL (2014) Endothelial NLRP3 inflammasome activation and enhanced neointima formation in mice by adipokine visfatin. Am J Pathol 184:1617-1628

Xiao H, Lu M, Lin TY, Chen Z, Chen G, Wang WC, Marin T, Shentu TP, Wen L, Gongol B, et al. (2013) Sterol regulatory element binding protein 2 activation of NLRP3 inflammasome in endothelium mediates hemodynamic-induced atherosclerosis susceptibility. Circulation 128:632-642.
Xie W and Tian Y (2006) Xenobiotic receptor meets NF-kappaB, a collision in the small bowel. Cell Metab 4:177-178.

Yang H, Xiao L, Yuan Y, Luo X, Jiang M, Ni J, and Wang N (2014) Procyanidin B2 inhibits NLRP3 inflammasome activation in human vascular endothelial cells. Biochem Pharmacol 92:599-606.

Yokode M (2001) Oxidized LDL and vascular endothelial dysfunction. Nihon Rinsho 59 (Suppl 2):596-601.

Zhang H, Park Y, Wu J, Chen Xp, Lee S, Yang J, Dellsperger KC, and Zhang C (2009) Role of TNF-alpha in vascular dysfunction. Clin Sci (Lond) 116:219-230.

Zhou C, Tabb MM, Nelson EL, Grün F, Verma S, Sadatrafiei A, Lin M, Mallick S, Forman BM, Thummel KE, et al. (2006) Mutual repression between steroid and xenobiotic receptor and NF-kappaB signaling pathways links xenobiotic metabolism and inflammation. $J$ Clin Invest 116:2280-2289.

Zhou C, Verma S, and Blumberg B (2009) The steroid and xenobiotic receptor (SXR), beyond xenobiotic metabolism. Nucl Recept Signal 7:e001.

Address correspondence to: Dr. Nanping Wang, The Advanced Institute for Medical Sciences, Dalian Medical University, Dalian 116044, People's Republic of China. E-mail: nanpingwang2003@yahoo.com or Dr. Lei Xiao, Cardiovascular Research Center, Xi'an Jiaotong University, Xi'an 710061, People's Republic of China. E-mail: xiaolei0122@xjtu.edu.cn 